\title{
$O$ pré-natal e a assistência de enfermagem à gestante de alto risco
}

\author{
Prenatal care and nursing care for high-risk pregnant women \\ Atención prenatal y atención de enfermería para mujeres embarazadas de alto riesgo
}

Mariana Pereira Barbosa Silva ORCID: https://orcid.org/0000-0003-0852-8099 Universidade Estadual do Piaú, Brasil E-mail: marianapbsilvaa@gmail.com

Iara Lima de Andrade Ferreira ORCID: https://orcid.org/0000-0002-7478-8553

Universidade Estadual do Piauí, Brasil E-mail: iaraferreira754@gmail.com

Samuel Lopes dos Santos

ORCID: https://orcid.org/0000-0003-3375-9171 Universidade Federal do Piauí, Brasil

E-mail: samuellopes121314@gmail.com Airton César Leite ORCID: https://orcid.org/0000-0001-7184-8488

Centro Universitário Santo Agostinho, Brasil E-mail: ainton.cesar2014@gmail.com

Maria Vitalina Alves de Sousa ORCID: https://orcid.org/0000-0003-4448-2489 Centro Universitário Inta, Brasil E-mail: enf.vitalinaalves@gmail.com

Bruno Abilio da Silva Machado ORCID: https://orcid.org/0000-0003-1759-0206 Centro Universitário Maurício de Nassau, Brasil E-mail: brunnoabillio92@gmail.com

Layanne Cavalcante de Moura ORCID: https://orcid.org/0000-0003-2781-1076 Centro Universitário Unifacid, Brasil

E-mail: layannecavalcante@hotmail.com

Gabriel Oliveira da Silva

ORCID: https://orcid.org/0000-0003-3261-0535

Centro Universitário Uninovafapi, Brasil

E-mail: enf.coisas@gmail.com

Mariane Rangel Gomes Campos ORCID: https://orcid.org/0000-0001-6000-8395

Universidade Estácio de Sá, Brasil

E-mail: marianergc@gmail.com

Nathália Menezes Dias

ORCID: https://orcid.org/0000-0002-2166-5293 Universidade Estadual do Pará, Brasil

E-mail: menezesdiasnathalia@gmail.com

Moacir Andrade Ribeiro Filho

ORCID: https://orcid.org/0000-0003-1991-469X Universidade Regional do Cariri, Brasil E-mail: moacirarf@outlook.com

Renata Laís Lima Silva

ORCID: https://orcid.org/0000-0001-8428-4247 Universidade Unopar, Brasil

E-mail: renata.lays45@gmail.com

Gustavo Baroni Araujo

ORCID: https://orcid.org/0000-0002-3162-7477

Universidade Estadual de Londrina, Brasil E-mail: gustavobaroni13@hotmail.com

Joelma Maria dos Santos da Silva Apolinário

ORCID: https://orcid.org/0000-0001-9521-9432

Faculdade Maurício de Nassau, Brasil

E-mail: jo.silva00@hotmail.com

Maria Dhescyca Ingrid Silva Arruda

ORCID: https://orcid.org/0000-0002-9073-7844

Faculdade São Francisco da Paraíba, Brasil E-mail: dhescycaingrid20@gmail.com 


\title{
Angelica Ribeiro do Nascimento Oliveira \\ ORCID: https://orcid.org/0000-0002-7240-2004 \\ Centro Universitário Maurício de Nassau, Brasil E-mail: angelicalribeiro.ar19@gmail.com
}

\begin{abstract}
Resumo
A gestação é uma fase importante na vida da mulher, porém pode causar riscos para a gestante e feto quando se trata de uma gravidez de alto risco. A gravidez é considerada de alto risco quando existe a possibilidade de um resultado adverso para a mulher e quando há presença de fatores ou determinantes de risco, e que, portanto, podem causar problemas de saúde ou até mesmo a morte materna e fetal. Este estudo tem como objetivo discutir a partir de achados na literatura a importância do pré-natal e a assistência de enfermagem à gestante de alto risco. Trata-se de um estudo descritivo, do tipo revisão integrativa de literatura, de caráter qualitativo, a busca dos artigos foi realizada com o auxílio das bases de dados LILACS, SciELO, BDENF, via BVS, e Scholar Google. Os artigos foram coletados no período de maio de 2021. Foram utilizados os descritores: "Pré-natal" and "Assistência de Enfermagem" and "Gravidez de alto risco", cruzados com o operador booleando "AND", localizados na lista dos Descritores em Ciências da Saúde (DECs). Após a aplicação dos critérios de elegibilidade, restaram 12 estudos que se adequaram ao objetivo proposto pela pesquisa. O enfermeiro desempenha um papel de extrema importância para que ocorra um prénatal de qualidade, devendo, portanto, prestar uma assistência humanizada, atendendo as queixas da paciente, executando e prescrevendo cuidados, a fim de diminuir os riscos e as possíveis complicações para a gestante de risco e ao feto. Conclui-se que o pré-natal e a assistência de enfermagem à gestante de alto risco é fundamental na redução de riscos e eventos adversos para a mãe e feto, contribuindo na redução da mortalidade de ambos, e melhorando o prognóstico das gestantes de alto risco, sendo fundamental um acompanhamento efetivo e eficaz às mesmas.
\end{abstract}

Palavras-chave: Pré-natal; Assistência de enfermagem; Gravidez de alto risco.

\begin{abstract}
Pregnancy is an important stage in a woman's life, but it can pose risks for the pregnant woman and fetus when it comes to a high-risk pregnancy. Pregnancy is considered high risk when there is a possibility of an adverse outcome for the woman and when there is the presence of risk factors or determinants, which, therefore, can cause health problems or even maternal and fetal death. This study aims to discuss, based on findings in the literature, the importance of prenatal care and nursing care for high-risk pregnant women. This is a descriptive study, of the integrative literature review type, with a qualitative character, the search for articles was performed with the help of the LILACS, SciELO, BDENF, via BVS, and Scholar Google databases. The articles were collected in the period of May 2021. The following descriptors were used: "Prenatal" and "Nursing Care" and "High-risk pregnancy", crossed with the operator booleaning "AND", located in the list of Descriptors in Health Sciences (DECs). After applying the eligibility criteria, 12 studies were left that fit the objective proposed by the research. The nurse plays an extremely important role for a quality prenatal care to occur, and must therefore provide humanized care, attending to the patient's complaints, executing and prescribing care, in order to reduce the risks and possible complications for the pregnant woman risk and the fetus. It is concluded that prenatal care and nursing care for high-risk pregnant women is essential to reduce risks and adverse events for the mother and fetus, contributing to the reduction of mortality for both, and improving the prognosis of high-risk pregnant women, being essential an effective and efficient follow-up to them.
\end{abstract}

Keywords: Prenatal; Nursing assistance; High-risk pregnancy.

\section{Resumen}

El embarazo es una etapa importante en la vida de una mujer, pero puede presentar riesgos para la mujer embarazada y el feto cuando se trata de un embarazo de alto riesgo. El embarazo se considera de alto riesgo cuando existe la posibilidad de un desenlace adverso para la mujer y cuando existe la presencia de factores de riesgo o determinantes, que, por tanto, pueden ocasionar problemas de salud o incluso la muerte materna y fetal. Este estudio tiene como objetivo discutir, con base en los hallazgos de la literatura, la importancia de la atención prenatal y la atención de enfermería para las mujeres embarazadas de alto riesgo. Se trata de un estudio descriptivo, del tipo revisión integradora de la literatura, con carácter cualitativo, la búsqueda de artículos se realizó con la ayuda de las bases de datos LILACS, SciELO, BDENF, vía BVS y Scholar Google. Los artículos fueron recolectados en el período de mayo de 2021. Se utilizaron los siguientes descriptores: "Prenatal" y "Atención de enfermería" y "Embarazo de alto riesgo", cruzados con el operador booleano que significa "AND", ubicado en la lista de Descriptores en Salud Ciencias (DECs). Después de aplicar los criterios de elegibilidad, quedaron 12 estudios que se ajustan al objetivo propuesto por la investigación. La enfermera juega un papel sumamente importante para que se produzca una atención prenatal de calidad, por lo que debe brindar una atención humanizada, atendiendo las quejas de la paciente, ejecutando y prescribiendo cuidados, con el fin de reducir los riesgos y posibles complicaciones para la gestante y el feto. Se concluye que la atención prenatal y de enfermería a la gestante de alto riesgo es fundamental para reducir los riesgos y eventos adversos para la madre y el feto, contribuyendo a la reducción de la mortalidad de ambos y mejorando el pronóstico de la gestante de alto riesgo, siendo fundamental un seguimiento eficaz y eficiente de los mismos.

Palabras clave: Prenatal; Asistencia de enfermería; Embarazo de alto riesgo. 


\section{Introdução}

A gestação é uma fase importante na vida da mulher, contudo pode ser um período de sofrimento quando não é desejável, ou quando eventos adversos comprometem a gravidez causando problemas de saúde ou até mesmo a morte materna (Medeiros et al., 2019).

Apesar de ser um processo fisiológico para a reprodução humana, a gestação pode causar riscos para a gestante e feto. A gravidez é considerada de alto risco quando existe a possibilidade de um resultado adverso para a mulher e quando há presença de fatores ou determinantes de risco. Esses riscos podem estar relacionados a doenças preexistentes ou intercorrências na gravidez por causas orgânicas, biológicas, químicas e ocupacionais, bem como devido a condições sociais e demográficas desfavoráveis. No Brasil, a prevalência de gestações de alto risco é imprecisa e, geralmente está relacionada a quadros de hipertensão arterial, infecções e diabetes gestacional (Ericco, Bicalho, Oliveira \& Martins, 2018).

O Ministério da Saúde (MS) implantou no ano de 2000, o Programa de Humanização no Pré-Natal e Nascimento (PHPN), garantindo assim a redução dos óbitos maternos e perinatais e uma assistência de qualidade e humanizada desde o parto até o puerpério (Lima et al., 2019). Nesse programa, toda gestante tem o direito de atendimento humanizado, seguro e de qualidade na gestação, parto e puerpério de acordo as condições estabelecidas pela prática médica; e, todo recém-nascido tem direito à assistência neonatal humanizada e segura. Para que isso ocorra deve-se ter consultas gestacionais e pediátricas, exames laboratoriais, exames de imagem, imunização, classificação de risco gestacional e atividades educativas para as gestantes (Cardoso et al., 2019).

A assistência para a gestante considerada de risco é um desafio no dia a dia da atenção à saúde, pois os altos índices de mortalidade materna preocupam não só a sociedade, mas também as autoridades. Salienta-se que o cuidado deve iniciar no pré-natal, o que corrobora em uma ferramenta de detecção precoce de fatores de risco que podem transformar uma gravidez de risco habitual em alto risco, possibilitando a intervenção em tempo oportuno para aqueles que são modificáveis (Ferreira et al., 2019).

Para um pré-natal de qualidade, a acessibilidade às unidades especializadas de referência vinculadas ao Sistema Único de Saúde (SUS) deve ser eficiente para atender as necessidades e particularidades das mulheres que desenvolvem gestação de alto risco. $\mathrm{O}$ início do pré-natal precoce e rápido referenciamento, quando identificado o risco, permite atender às singularidades das gestantes e prestar o cuidado de saúde com qualidade. Porém, ainda é alto o número de gestantes que descobrem a gravidez em estágio mais avançado, iniciando assim o acompanhamento na rede de atenção em saúde tardiamente, após o primeiro trimestre de gestação (Guerra, Valete \& Alves, 2019). No entanto, os cuidados assistenciais no primeiro trimestre são considerados um indicador da qualidade dos cuidados maternos e fundamentais para um bom prognóstico gestacional (Medeiros et al., 2019).

O acompanhamento pré-natal inicia-se na Unidade Básica de Saúde (UBS) de referência de cada bairro e respectivo município de residência da gestante. Na primeira consulta médica ou da enfermagem, a gestante é classificada como risco habitual, posteriormente o médico ou enfermeiro é responsável por verificar os antecedentes obstétricos, doenças ou agravos, condições individuais e sociodemográficas, condições clínicas pré-existentes ou alguma intercorrência clínica para continuar o acompanhamento no risco habitual ou possível seguimento para o risco intermediário ou alto risco (Costa et al., 2016).

A assistência pré-natal é uma estratégia recomendada para melhorar os resultados maternos e neonatais durante a gravidez, sendo fundamental para as gestantes com moderado a alto risco, pois ajuda no controle e prevenção de resultados adversos (Soares \& Higarashi, 2019).

O pré-natal tem como objetivo abordar, acolher e assegurar as necessidades da mulher no início até o final da gestação, garantindo o acompanhamento, o desenvolvimento e a observação de todos os aspectos da gestação, permitindo o parto de um recém-nascido saudável, sem impacto para a saúde materna, promovendo assim o bem-estar físico, mental e a 
saúde durante toda a gravidez. No entanto é fundamental identificar precocemente os danos que poderão resultar em maiores agravos à saúde da mãe e do bebê, possibilitando um cuidado diferenciado (Lima et al., 2019).

A assistência pré-natal é essencial para a redução de riscos, prevenção de doenças e promoção da saúde da mulher e da criança, tem como principal objetivo identificar esses possíveis riscos e proporcionar o cuidado com o manejo adequado dos mesmos, prevenindo assim as mortes maternas e infantis ou reduzindo os riscos de sua ocorrência (Garcia et al., 2019).

O acompanhamento do pré-natal de alto risco deve ser realizado por enfermeiros, que compõem a equipe multiprofissional, através de ações de prevenção e tratamento das morbidades que afetam a mãe e o feto, além de orientações quanto o parto normal, amamentação e puerpério (Jorge, Silva \& Makuch, 2020).

A gravidez de alto risco é uma experiência estressante, portanto, é fundamental o adequado acolhimento e assistência a esta gestante tanto psicológica quanto física, necessitando de uma equipe preparada, compreensiva e paciente (Santos et al., 2016). Uma assistência qualificada voltada a essas mulheres, principalmente a assistência pré-natal, pode mudar os prognósticos tanto para a mãe quanto para o feto, contribuindo para o aumento de desfechos favoráveis (Teles et al., 2019).

Diante disso, este estudo tem como objetivo discutir a partir de achados na literatura a importância do pré-natal e a assistência de enfermagem à gestante de alto risco.

\section{Metodologia}

Trata-se de um estudo descritivo, do tipo revisão integrativa de literatura, de caráter qualitativo. Segundo Mendes, Silveira, \& Galvão (2008) esse tipo de revisão inclui a análise de pesquisas relevantes que dão suporte para a tomada de decisão e a melhoria da prática clínica, permitindo a síntese do conhecimento de um determinado assunto, além disso, permite a síntese de múltiplos estudos publicados possibilitando conclusões gerais a respeito de uma área de estudo específica.

A revisão integrativa é uma ferramenta de investigação que permite a procura, a avaliação crítica e a síntese de evidências disponíveis sobre o tema investigado, em que o produto final é o estado do conhecimento, a implementação de intervenções efetivas na prestação de cuidados e na redução de custos. Além disso, permite a identificação de fragilidades, que poderão conduzir ao desenvolvimento de futuras investigações (Sousa, Marques-Vieira, Severino, \& Antunes, 2017).

A revisão da literatura serve para reconhecer a unidade e a diversidade interpretativa existente no eixo temático em que se insere o problema em estudo, para ampliar, ramificar a análise interpretativa, bem como para compor as abstrações e sínteses que qualquer pesquisa requer colaborando para a coerência nas argumentações do pesquisador. Nesse sentido, a revisão integrativa é um método que tem como finalidade reunir e sintetizar resultados de pesquisas sobre um delimitado tema ou questão, de maneira sistemática e ordenada e abrangente, contribuindo para o aprofundamento do conhecimento do tema investigado (Ferenhof \& Fernandes, 2016).

Foi seguida as seguintes etapas: definição do tema e elaboração da questão de pesquisa; elaboração dos critérios de elegibilidade, inclusão e exclusão dos estudos; definição dos descritores, busca na literatura e coleta de dados; análise crítica dos estudos incluídos e discussão dos resultados; e apresentação da síntese da revisão.

Para direcionar a presente revisão delineou-se como questão norteadora: "O que a literatura aborda sobre a importância do pré-natal e a assistência de enfermagem à gestante de alto risco?”.

Para a construção deste trabalho, a busca dos artigos foi realizada na Biblioteca Virtual em Saúde (BVS), com o auxílio das bases de dados Literatura Latino-Americana e do Caribe em Ciências da Saúde (LILACS), Scientific Electronic Library Online (SciELO), Base de Dados de Enfermagem (BDENF) e Scholar Google. Os artigos foram coletados no período de maio de 2021. Foram utilizados os descritores: "Pré-natal" and "Assistência de Enfermagem" and "Gravidez de alto risco", cruzados com o operador booleando “AND”, localizados na lista dos Descritores em Ciências da Saúde (DECs). 
Foram selecionados como critérios de inclusão: artigos completos disponíveis na íntegra, nos idiomas português, espanhol e inglês, com recorte temporal de 2016 a 2021. E os critérios de exclusão foram: artigos incompletos, duplicados, debates, resenhas, resumos ou artigos publicados em anais de eventos, indisponíveis na íntegra.

Ressalta-se que, quanto aos aspectos éticos, por se tratar de um estudo de revisão, não foi necessário o encaminhamento e aprovação de Comitê de Ética em Pesquisa. No entanto, foram respeitados os preceitos éticos e a garantia dos direitos autorais das obras utilizadas.

\section{Resultados e Discussão}

A seguir, o fluxograma embasado no Preferred reporting items for systematic reviews and meta-analyses (PRISMA) sintetiza a busca dos artigos que compuseram a amostra final da revisão (Figura 1).

Figura 1: Fluxograma com o processo de seleção dos artigos que compuseram a síntese final.

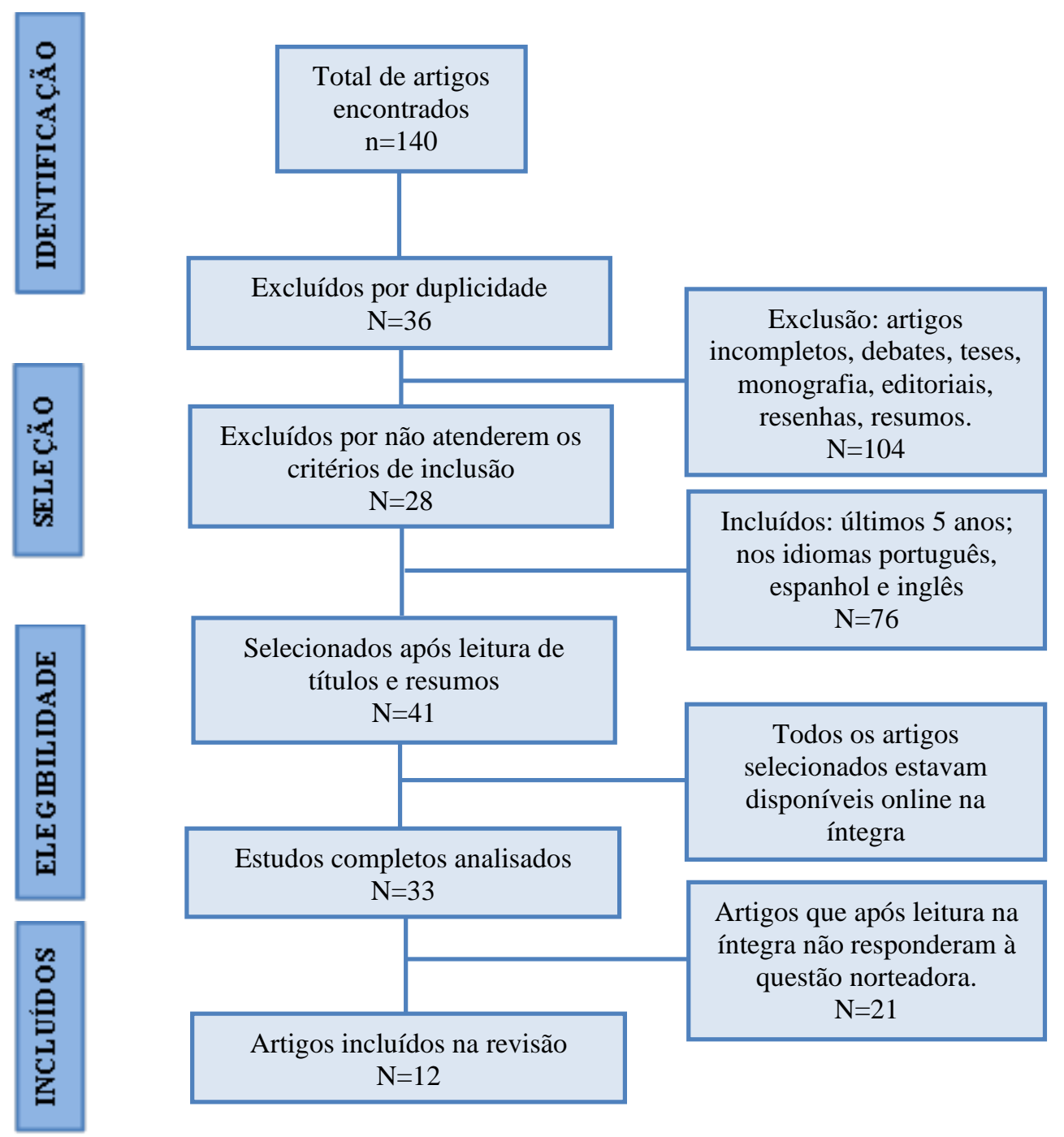

Fonte: Elaborado pelos autores a partir do Fluxograma PRISMA (2021). 
Na figura 1 observa-se que a partir da coleta de dados, localizaram-se 140 estudos, dos quais 36 foram excluídos por duplicidade e 28 por não atenderem aos critérios de inclusão, restando 76 artigos, que após leitura dos títulos e resumos obteve-se uma amostra de 33 estudos, na primeira etapa da avaliação.

Na segunda etapa, procedeu-se a leitura dos 33 estudos, desses, 21 foram excluídos por não responder à questão norteadora. Ao final foram incluídos 12 estudos que se adequaram ao objetivo proposto pela pesquisa.

Diante disso, os estudos incluídos para análise final foram organizados em uma planilha de dados contendo as seguintes variáveis: autor, título, ano e metodologia empregada (Quadro 1).

Quadro 1. Caracterização dos artigos conforme autor, título, ano e metodologia.

\begin{tabular}{|c|c|c|c|c|}
\hline $\mathbf{N}^{\mathbf{o}}$ & Autor & Título & Ano & Metodologia \\
\hline 1 & $\begin{array}{l}\text { Jorge, Silva \& } \\
\text { Makuch }\end{array}$ & $\begin{array}{l}\text { Assistência humanizada no } \\
\text { pré-natal de alto risco: } \\
\text { percepções de enfermeiros }\end{array}$ & 2020 & Estudo qualitativo \\
\hline 2 & Lima et al. & $\begin{array}{l}\text { Assistência de Enfermagem } \\
\text { no Pré-Natal de Alto risco }\end{array}$ & 2019 & Revisão de literatura \\
\hline 3 & Júnior et al. & $\begin{array}{l}\text { O enfermeiro no pré-natal de } \\
\text { alto risco: papel profissional. }\end{array}$ & 2017 & $\begin{array}{l}\text { Estudo exploratório descritivo, } \\
\text { com abordagem qualitativa }\end{array}$ \\
\hline 4 & $\begin{array}{l}\text { Ericco, Bicalho, } \\
\text { Oliveira \& Martins }\end{array}$ & $\begin{array}{l}\text { O trabalho do enfermeiro no } \\
\text { pre-natal de alto risco sob a } \\
\text { ótica das necessidades } \\
\text { humanas básicas. }\end{array}$ & 2018 & $\begin{array}{c}\text { Estudo transversal, descritivo e de } \\
\text { abordagem quantitativa }\end{array}$ \\
\hline 5 & Medeiros et al. & $\begin{array}{l}\text { Acompanhamento pré-natal } \\
\text { da gestação de alto risco no } \\
\text { serviço publico. }\end{array}$ & 2019 & $\begin{array}{l}\text { Estudo transversal analítico, de } \\
\text { abordagem quantitativa }\end{array}$ \\
\hline 6 & $\begin{array}{l}\text { Carpes, Biff, \& } \\
\text { Stumm }\end{array}$ & $\begin{array}{l}\text { Percepção de acadêmicos de } \\
\text { enfermagem acerca do papel } \\
\text { do enfermeiro no cuidado } \\
\text { pré-natal. }\end{array}$ & 2019 & $\begin{array}{c}\text { Estudo descritivo com abordagem } \\
\text { qualitativa }\end{array}$ \\
\hline 7 & Santos et al. & $\begin{array}{l}\text { Qualidade da assistência de } \\
\text { enfermagem prestada à } \\
\text { gestante de alto risco em } \\
\text { âmbito hospitalar. }\end{array}$ & 2016 & $\begin{array}{c}\text { Estudo descritivo, de caráter } \\
\text { observacional e abordagem } \\
\text { qualitativa }\end{array}$ \\
\hline 8 & $\begin{array}{c}\text { Oliveira, Barbosa \& } \\
\text { Melo }\end{array}$ & $\begin{array}{l}\text { A importância do } \\
\text { acompanhamento pré-natal } \\
\text { realizado por enfermeiros. }\end{array}$ & 2016 & $\begin{array}{c}\text { Revisão bibliográfica, descritiva e } \\
\text { exploratória }\end{array}$ \\
\hline 9 & Ferreira et al. & $\begin{array}{l}\text { Cuidado de enfermagem na } \\
\text { ótica das gestantes de alto } \\
\text { risco. }\end{array}$ & 2019 & $\begin{array}{c}\text { Estudo descritivo, com abordagem } \\
\text { qualitativa }\end{array}$ \\
\hline 10 & Rocha \& Andrade & $\begin{array}{l}\text { Atenção da equipe de } \\
\text { enfermagem durante o pré- } \\
\text { natal: percepção das } \\
\text { gestantes atendidas na rede } \\
\text { básica de Itapuranga-GO em } \\
\text { diferentes contextos sociais }\end{array}$ & 2017 & $\begin{array}{c}\text { Estudo observacional, transversal e } \\
\text { qualiquantitativo }\end{array}$ \\
\hline 11 & Costa et al. & $\begin{array}{l}\text { Adequação do pré-natal de } \\
\text { alto risco em um hospital de } \\
\text { referência }\end{array}$ & 2016 & $\begin{array}{c}\text { Pesquisa descritiva, retrospectiva e } \\
\text { documental }\end{array}$ \\
\hline 12 & Ferreira et al., & $\begin{array}{l}\text { Pré-natal e a atuação dos } \\
\text { profissionais da enfermagem } \\
\text { em prol da saúde da gestante } \\
\text { e do recém-nascido. }\end{array}$ & 2019 & Revisão integrativa da literatura \\
\hline
\end{tabular}


O enfermeiro desempenha um papel de extrema importância para que ocorra um pré-natal de qualidade, devendo, portanto a equipe de enfermagem estar apta a realizar uma assistência humanizada, baseada na atenção às queixas da paciente, executando e prescrevendo cuidados, orientações de qualidade durante o atendimento, a fim de assegurar uma gestação sem intercorrências ou minimizando os agravos/desconfortos que podem surgir no decorrer da gestação (Rocha \& Andrade, 2017).

A assistência durante o pré-natal permite a avaliação de possíveis fatores de risco e a identificação de problemas e agravos, impedindo assim um resultado desfavorável à ocasião do ciclo gravídico. É importante destacar que uma gestação de alto risco pode se configurar a qualquer momento, sendo necessário haver uma reclassificação quanto ao grau de risco que cada gestante tem em sua consulta de pré-natal e também durante o trabalho de parto (Lima et al., 2019).

Um pré-natal ideal deve ter no mínimo seis consultas, devendo iniciar no primeiro trimestre, duas no segundo e três no terceiro trimestre de gestação. Os intervalos entre consultas devem ser de quatro semanas até que complete 36 semanas. Após os intervalos são de 15 dias. Em casos de gestantes faltosas a equipe de enfermagem juntamente com os agentes comunitários de saúde, deve realizar busca ativa dessas gestantes (Rocha \& Andrade, 2017).

$\mathrm{Na}$ atenção pré-natal de alto risco (PNAR) o Ministério da Saúde preconiza o atendimento da gestante por equipe multidisciplinar, que inclui o profissional enfermeiro. Dentre as ações do enfermeiro destaca-se a consulta de enfermagem que permite identificar os problemas reais e potenciais da gestante e, consequentemente, elaborar o planejamento das ações de cuidado necessárias. A consulta é o momento onde se reafirma a singularidade da mulher e inicia-se o processo de compartilhamento das responsabilidades com a pactuação das metas (Ericco, Bicalho, Oliveira \& Martins, 2018).

A consulta de enfermagem é uma atividade independente, realizada privativamente pelo enfermeiro e objetiva proporcionar condições para a promoção da saúde da gestante. O principal papel do enfermeiro nessas atividades é: orientar as mulheres e suas famílias sobre a importância do pré-natal, da amamentação e da alimentação; desenvolver atividades educativas, em grupos e individuais visando unir as gestantes trazendo-as para perto do enfermeiro, assim como a família e a comunidade; identificar as gestantes com algum sinal de alerta como identificadas de alto risco e encaminhá-las para consultas médicas o mais rápido possível; realizar consultas de pré-natal, solicitar exames conforme protocolo local de pré natal; realizar exames físicos como testes das mamas e coleta de citopatológico do colo do útero (caso necessário); e fazer com que a gestante crie um vínculo de confiança, para que o processo da assistência se dê de forma transparente (Carpes, Biff, \& Stumm, 2019).

A consulta de enfermagem é importante, pois permite um contato direto com o paciente, permitindo uma melhor compreensão da patologia ou situação de saúde que o acomete, assim podendo desenvolver uma assistência de enfermagem adequada (Júnior et al., 2017). A consulta juntamente com a promoção de um ambiente de segurança e confiança durante os cuidados de preconcepção, pré-natal, intraparto e pós-natal, contribuem para a melhoria da saúde e bem-estar da mãe e do feto (Jorge, Silva \& Makuch, 2020).

A gestante de risco deve ser encaminhada ao serviço de referência, tendo o cuidado garantido no estabelecimento de origem no momento do encaminhamento até o final da gestação, com o trânsito facilitado entre os serviços de saúde, assegurando atendimento adequado em tempo conveniente. A avaliação de risco deve ocorrer individualmente durante a anamnese, com reavaliação do risco gestacional em todas as consultas do pré-natal (Medeiros et al., 2019).

A periodicidade das consultas é determinada pela equipe responsável, de acordo com as necessidades e prioridades de cada gestante. A equipe de saúde, que realiza o acompanhamento da gestação de alto risco, deve considerar: avaliação clínica, avaliação obstétrica, repercussões entre as condições clínicas da gestante e a gravidez, determinação da via de parto, aspectos emocionais e psicossociais. Os principais cuidados realizados no pré-natal estão direcionados ao exame físico, sendo eles, peso materno, pressão arterial (PA), altura uterina (AU) e ausculta dos batimentos cardíacos fetais (BCF) (Medeiros et al., 2019).

Assistir as mulheres com maior risco gestacional é permitir compreender e apreender, a partir de experiências individuais e coletivas, a vivência de eventos patológicos ou não aos quais se encontram expostas, e que podem gerar 
vulnerabilidades a serem consideradas na boa assistência pré-natal. A assistência de enfermagem precisa estar direcionada a amparar e focar o cuidado de forma a contribuir para a segurança, a tranquilidade e o enfrentamento da gestante quanto à situação em que ela se encontra (Ferreira et al., 2019).

O acesso à unidade de saúde é essencial para o acolhimento na atenção primária, onde a gestante deve receber um atendimento humanizado. O enfermeiro é o profissional que está à frente da coordenação e é responsável por gerenciar todo e qualquer atendimento. É importante que o registro nos prontuários das pacientes seja cuidadosamente gerenciado pelos profissionais, visto que o mesmo indica as ações realizadas e permite a continuidade da assistência ofertada, comprovando o atendimento prestado, a ausência de informações dos exames solicitados e registro dos resultados podem ser avaliados como imprudência, negligência ou imperícia (Costa et al., 2016).

O acompanhamento durante o pré-natal é de grande valia, sendo importante que a gestante participe desse programa de apoio, principalmente porque as intervenções dos profissionais da saúde durante esse período, em promoção da saúde materna, podem prevenir riscos e, principalmente, garantir suporte para o fortalecimento físico, nutricional e emocional para a mulher grávida (Ferreira et al., 2019).

A equipe de enfermagem que presta assistência a estas gestantes de risco deve estar preparada para diminuir os riscos e as possíveis complicações para mulher e feto. Para isso é fundamental que os profissionais tenham conhecimento também dos direitos da gestante durante a internação e o trabalho de parto. A mulher tem direito a ser escutada, ter o esclarecimento de suas dúvidas e expressar-se livremente, o que pressupõe que a equipe de enfermagem deve ser treinada para atender e não ignorar estas necessidades (Santos et al., 2016).

O enfermeiro não necessita apenas de sua competência técnica, mas também da escuta qualificada, ouvindo as queixas, preocupações e angústias da gestante, criando, assim, uma relação mais próxima com a mesma, sua família e comunidade (Oliveira, Barbosa \& Melo, 2016).

O papel do enfermeiro consiste em realizar avaliação psicossocial e nutricional, educação em saúde, aconselhamento perinatal, apoio na gestão do serviço e tomada de decisões. Dentre as ações de educação em saúde, destacam-se as orientações sobre as alterações fisiológicas da gravidez, o uso dos métodos não farmacológicos para o alívio da dor durante o parto, crescimento e desenvolvimento fetal e amamentação (Jorge, Silva \& Makuch, 2020).

No estudo realizado por Medeiros et al. (2019) acredita-se que a realização de grupos de gestantes pode ser um meio valioso de orientação, pois o enfermeiro, ao elaborar atividades em grupo, consegue atingir um número importante de mulheres, sendo esta estratégia educativa benéfica para a saúde da gestante, contribuindo positivamente para a eficácia do prénatal.

A educação em saúde no pré-natal permite a preparação da mulher para a gestação e o parto, porém as mulheres ainda enfrentam dificuldades quanto ao acesso ao pré-natal, à escassez de profissionais para compor a equipe multidisciplinar e carência de estrutura institucional (Jorge, Silva \& Makuch, 2020).

A educação em saúde na gestação de alto risco é fundamental, e a enfermagem, sendo a profissão voltada para o cuidado, deve repassar conhecimento científico, de maneira informal, para que as gestantes possam compreender e realizar reflexões sobre o que foi discutido, desenvolvendo uma gestação saudável e tranquila (Júnior et al., 2017).

O enfermeiro deve transmitir as orientações de forma compreensiva, adequada às reais necessidades de cada gestante, oferecendo subsídio para a adoção de novos hábitos e condutas de saúde, o que possibilita o autocuidado, desde que as gestantes sejam participativas e não apenas receptoras de informações (Júnior et al., 2017).

Os profissionais de saúde devem desempenhar uma assistência de qualidade e satisfatória, respeitando as pessoas em seus aspectos econômicos, culturais, espirituais e biopsicossociais, estimulando-as a procurarem o serviço de saúde (Júnior et al., 2017). 
O profissional de enfermagem deve, portanto estar capacitado para exercer seu papel de forma efetiva, de maneira evidente e clara, retirando dúvidas e promovendo ações de forma integral como a explicação de alterações fisiológicas e psicológicas que as mulheres passam ter neste momento de sua vida (Lima et al., 2019). E ainda, torna-se importante o trabalho em equipe, com compartilhamento substantivo das ações e efetiva negociação, com a gestante, de seu cuidado (Ericco, Bicalho, Oliveira \& Martins, 2018).

Desse modo, a enfermagem, por estar diretamente e integralmente assistindo essas mulheres, tem um papel fundamental na garantia do seu bem-estar, que está integrado no diálogo, na confiança e no conforto. O apoio emocional, o estímulo à verbalização e à expressão dos sentimentos e vivências e a promoção da educação em saúde possibilitará a elas a vivência do processo de adoecimento de forma mais tranquila e confiante (Ferreira et al., 2019).

\section{Conclusão}

Esse estudo é relevante à medida que proporciona conhecer a importância do pré-natal e a assistência de enfermagem à gestante de alto risco. Observa-se que o pré-natal é fundamental na redução de riscos e eventos adversos para a mãe e feto, contribuindo na redução da mortalidade de ambos, e melhorando o prognóstico principalmente de gestantes de alto risco, sendo fundamental um acompanhamento efetivo e eficaz às mesmas.

A equipe de enfermagem é essencial para um pré-natal de qualidade, no entanto para que isso ocorra é importante que o profissional esteja atento às demandas da paciente, tirando suas dúvidas e prestando uma assistência integral e acolhedora, a fim de oferecer um suporte psicológico diante da situação estressante em que a gestante se encontra.

E ainda, ressalta-se a necessidade dos profissionais se capacitarem para promover esclarecimentos e promover o atendimento necessário às pacientes e aos familiares, a fim de prestar uma assistência digna e de qualidade, permitindo através de grupos, rodas de conversas, e por meio da educação em saúde, estratégias para inserção das gestantes acerca da importância do pré-natal e acompanhamento da gestação de risco.

Destarte, torna-se excepcional a realização de novas pesquisas com a finalidade de ampliar a produção científica e promover discussões quanto ao pré-natal e a assistência de enfermagem à gestante de alto risco.

\section{Referências}

Cardoso, S. L., Souza, M. E. V., Oliveira, R. S., Souza, A. F., Lacerda, M. D. F., Oliveira, N. T. C., Castro, A. P. R., \& Medeiros, K. M. F. (2019). Ações de promoção para saúde da gestante com ênfase no pré-natal. Rev Interfaces Saúde Hum Tecnol, 7(1), 180-186.

Carpes, F., Biff, D., \& Stumm, K. E. (2019). Percepção de acadêmicos de enfermagem acerca do papel do enfermeiro no cuidado pré-natal. Revista Enfermagem Atual In Derme, 79(17), 20-22.

Costa, L. D., Perondi, A. R., Cavalheiri, J. C., Ferreira, A. S., Teixeira, G. T., \& Bortoloti, D. S. (2016). Adequação do pré-natal de alto risco em um hospital de referência. Rev Rene, 17(4),459-465.

Errico, L. S. P., Bicalho, P. G., Oliveira, T. C. F. L., \& Martins, E. F. (2018). O trabalho do enfermeiro no pre-natal de alto risco sob a ótica das necessidades humanas básicas. Rev Bras Enferm, 71(Suppl 3), 1257-64.

Ferenhof, H. A., \& Fernandes, R. F. (2016). Desmistificando a revisão de literatura como base para redação científica: método SSF. Revista ACB, 21(3), 550563.

Ferreira, M. A., Justo, F. K. M., Nicolau, M. S., Vieira, M. J. C., Sousa, R. R. G., \& Lima, F. M. S. (2019). Pré-natal e a atuação dos profissionais da enfermagem em prol da saúde da gestante e do recém-nascido. ID on line REVISTA DE PSICOLOGIA, 13(47), 764-772.

Ferreira, S. V., Soares, M. C., Cecagno, S., Alves, C. N., Soares, T. M., \& Braga, L. R. (2019). Cuidado de enfermagem na ótica das gestantes de alto risco. Revista Família, Ciclos de Vida e Saúde no Contexto Social, 7(2), 143-150.

Garcia, E. M., Martinelli, K. G., Gama, S. G. N., Oliveira, A. E., Esposti, C. D. D., \& Santos Neto, E. T. (2019). Risco Gestacional e Desigualdades Sociais: Uma Relação Possível?. Ciência \& Saúde Coletiva, 24(12), 4633-4642.

Guerra, J. V. V., Valete, C. O. S., \& Alves, V. H. (2019). Perfil sóciodemográfico e de saúde de gestantes em um pré-natal de alto risco. Braz. J. Hea. Rev., 2(1), 249-261.

Jorge, H. M. F., Silva, R. M., \& Makuch, M. Y. (2020). Assistência humanizada no pré-natal de alto risco: percepções de enfermeiros. Rev Rene, 21 , e44521. 
Research, Society and Development, v. 10, n. 9, e9410917173, 2021

(CC BY 4.0) | ISSN 2525-3409 | DOI: http://dx.doi.org/10.33448/rsd-v10i9.17173

Junior, A. R. F., Oliveira Filho, J. T., Rodrigues, M. E. N. G., Albuquerque, R. A. S., Siqueira, D. D. Á., \& Rocha, F. A. A. (2017). O enfermeiro no pré-natal de alto risco: papel profissional. Revista Baiana de Saúde Pública, 41(3), 650-667.

Lima, K. M. S. G., Santos, H. J., Pereira, J., Barbosa, L. P., Cabral, M. C. A. M., Silva, P. R., Santos, S. M. M., \& Souza, S. J. G. (2019). Assistência de Enfermagem no Pré-Natal de Alto risco. Braz. J. Hea. Rev., 2(4), 3183-3197.

Medeiros, F. F., Santos, I. D. L., Ferrari, R. A. P., Serafim, D., Maciel, S.M., \& Cardelli, A. A. M. (2019). Acompanhamento pré-natal da gestação de alto risco no serviço publico. Rev Bras Enferm,72(Suppl 3), 204-11.

Mendes, K. D. S., Silveira, R. C. C. P., \& Galvão, C. M. (2008). Revisão integrativa: método de pesquisa para a incorporação de evidências na saúde e na enfermagem. Texto \& Contexto - Enfermagem, 17(4), 758-764.

Oliveira, E. C., Barbosa, S. M., \& Melo, S. E. P. (2016). A importância do acompanhamento pré-natal realizado por enfermeiros. Revista Científica FacMais, $7(3), 24-38$

Rocha, A. C., \& Andrade, G. S. (2017). Atenção da equipe de enfermagem durante o pré-natal: percepção das gestantes atendidas na rede básica de Itapuranga-GO em diferentes contextos sociais. Revista Enfermagem Contemporânea, 6(1), 30-41.

Santos, M. B., Cardoso, S. M. M., Brum, Z. P., Rodrigues, A. P., Machado, N. C. B., \& Rocha, L. S. (2016). Qualidade da assistência de enfermagem prestada à gestante de alto risco em âmbito hospitalar. ScientiaTec, 3(2), 25-38.

Soares, L. G., \& Higarashi, I. H. (2019). Gestão de caso como estratégia de cuidado no pré-natal de alto risco. Revista Brasileira de Enfermagem, 72(3), 692699.

Sousa, L. M. M., Marques-Vieira, C. M. A., Severino, S. S. P., \& Antunes, A. V. (2017). A metodologia de revisão integrativa da literatura em enfermagem. Revista Investigação em Enfermagem, 21(2), 17-26.

Teles, P., Costa, E., Panobianco, M., Gozzo, T., Paterra, T., \& Nunes, L. (2019). Diagnósticos de enfermagem mais prevalentes em gestantes de alto risco. Enfermagem em Foco, 10(3), 119-125. 